\title{
Genome-Wide Identification, Characterization and Expression Analysis of Non-Arginine Aspartate Receptor like kinase gene family under Colletotrichum truncatum stress conditions in Hot pepper
}

R Srideepthi ${ }^{1}$, MSR Krishna ${ }^{1 *}$, Suneetha $\mathrm{P}^{2}$, R Sai Krishna ${ }^{3}$, Karthikeyan $\mathrm{S}^{1}$,

1: Department of Biotechnology, Koneru Lakshmaiah Education Foundation, Guntur, Andhra Pradesh, India

2: Institute of biotechnology, Professor Jaya Shankar Telangana State Agricultural University, Hyderabad, India

3: Crop production, ICAR-National Rice Research Institute, Cuttack, Odisha, India

1 *Corresponding author: msrkrishna81@gmail.com 


\section{Abstract}

3 Receptor Like kinases (RLKs) are conserved upstream signaling molecules that regulate several

4 biological processes, including plant development and stress adaptation. Non arginine aspartate

5 (non-RD) an important class of RLKs plays a vital role in disease resistance and apoptosis in

6 plants. In present investigation, a comprehensive Insilco analysis for non-RD Kinase gene family

7 including identification, sequence similarity, phylogeny, chromosomal localization, gene

8 structures, gene duplication analysis, promoter analysis and transcript expression profiles were

9 elucidated. In this study twenty six genes were observed on nine out of twelve chromosomes. All

10 these genes were clustered into seven subfamilies under large monophyletic group termed as

11 Interleukin-1 Receptor-Associated Kinase (IRAK) family. Structural diversity in genomic

12 structure among non-RD kinase gene family were identified and presence of pathogen induced

13 cis regulatory elements like STRE, MYC, MYB,W box were found. Expression profiles of genes

14 involved in providing resistance to anthracnose pathogen Colletotrichum truncatum in hot

15 pepper were analyzed at different infective stages in both resistant and susceptible genotypes.

16 Among twenty six genes, CaRLK1 gene belonging to LRRXII subfamily was up regulated under

17 severe stress after infection in resistant genotype PBC-80. This integrative approach has helped

18 us to identify candidate genes involved in disease resistance which would be helpful in future

19 crop improvement programs.

20 Keywords: Pattern Recognition Receptors (PRRs), Autophosphorylation, Downstream

21 Signaling, Colletotrichum truncatum, defense responses. 


\section{Introduction}

28 One of the major challenges in the $21^{\text {st }}$ century, to global food security and agricultural 29 sustainability is to develop economically important high yielding varieties which are stable with broad-spectrum of resistance. Hot pepper is a commercially important vegetable crop grown worldwide for its indispensable nutritional and therapeutic values, but year by year its

32 production has been reduced due to several biotic stresses [1]. Reason might be due to existence of limited resistant cultivars, existence of variability within the Colletotrichum truncatum species with erratic pathogenic ability with respect to different hosts, climatic conditions and commercial pesticides used remained as an unsatisfactory measures for its effective control [2]. As a part of its development of anthracnose resistant hot pepper remained as one of the major tasks to be resolved by modern agriculture practices prevailing worldwide. Congruently knowledge of understanding the defensive signaling mechanisms employed by resistant plants while encountering the attacking pathogen by advanced molecular and computational techniques paved a ray of hope to address these challenges [3].

RLKs are surface localized receptor-like protein kinases employed by Pattern recognition receptor (PRRs) proteins of plants innate immune system as a primary defensive response [4].

43 These are involved in signal perception from pathogen by ectodomain, then transduction of 44 signal by transmembrane region and then activation / deactivation of signaling cascade by kinase domain. Classification of ecto-domain was done based on the type of ligand binding specificities

46 [5] and endo domain based on presence or absence of conserved arginine residues present 47 immediately preceding to aspartate in catalytic domain VI of kinase domain [6]. Among various 48 types of RLKs, predominantly non-RD RLKs were allied with innate immune receptors that recognize conserved microbial signatures and activate pattern triggered immunity (PTI) involved 
50 in disease resistance [7]. Till now 35 genes in Arabidopsis and 328 genes in Rice were identified

51 to possess non-RD class of kinase receptor proteins. Functionally characterized non-RD kinases

52 like, XA21 BSR1 and XA26 from Rice and FLS2, EFR from Arabidopsis thaliana were known

53 to be effective against bacteria [8] while Pi-D2 gene of lectin (non-RD) kinase was found to

54 express broad-spectrum resistance against Magnaporthe grisea [9]. LecRK-VI.2 from

55 Arabidopsis thaliana [10] and WRKY from tomato [11] serve as a potential link in providing

56 resistance against bacterial and fungal pathogens. Hence due to limited availability of literature

57 on non-RD kinases in hot pepper and available free online software motivated us to identify the

58 presence of candidate non-RD genes associated with disease resistance through comparative

59 genomics.

60 Materials and methods

\section{Identification of transmembrane receptor kinases in hot pepper}

62 Identified and known RLKs from sequenced plant genomes like Arabidopsis, Rice and tomato

63 were collected as query sequences from NCBI (https://www.ncbi.nlm.nih.gov). Collected query

64 sequences were submitted to blast search against hot pepper CM-334 variety genome datasets

65 (http://www.solgenomics.net) version 1.55 of capsicum annuum. Protein sequences sharing more

66 than 50\% homology with query were selected for further analysis. The presence of signal peptide

67 was determined by SignalP 4.0 Server (http://www.cbs.dtu.dk/services/SignalP/)[12].

68 Extracellular ligand-binding domain, transmembrane, and intracellular kinase domain were

69 filtered by online domain search databases SMART (http://smart.emblheidelberg.de/) [13] and

70 Pfam with inbuilt HMMER search platform [14]. While Conserved motifs were identified using

71 the MEME program (http://meme-suite.org/index.html). This program was run with default 
72 settings except to set a maximum ten in number with the three hundred widths of motifs [15].

73 Based on the conserved motifs and phylogenetic analysis, receptors like kinases in hot pepper

74 were bundled.

\section{Phylogenetic analysis of transmembrane receptor kinases}

76 All known and recognized transmembrane receptor protein kinases (RLKs) belonging to model

77 plant species like Solanum Lycopersicum [16], Arabidopsis thaliana and Oryza sativa [17] were

78 taken together for phylogenetic analysis. Identified and characterized reference proteins at least

79 one from each family and subfamilies belonging to respective clades of three model plants along

80 with identified putative gene CaRLK Fasta sequences were submitted to MEGA software version

816.0 [18]. All sequences of CaRLK genes were submitted to multiple sequences alignment using

82 Multiple Sequence Comparison by Log- Expectation (MUSCLE) with default values. Then

83 phylogenetic tree was constructed using the Neighbor-joining (NJ) method with thousand

84 numbers of bootstrap replications.

\section{Analysis of CaRLK Physiochemical Properties}

Physiochemical parameters of putative CaRLK gene including molecular weight, isoelectric

87 point, number of amino acids, aliphatic index, and grand average of hydropathicity (GRAVY)

88 score was determined using online ExPASy programs (http://www.expasy.org/). Subcellular

89 locations of CaRLK proteins were predicted using the online Plant-PLoc tool

90 (http://www.csbio.sjtu.edu.cn/bioinf/plant-multi/) program. 
92 Information regarding position and location of chromosomes on which CaRLK gene members

93 were present was derived from hot Pepper Genome Platform (PGP) (http://Hot

94 peppergenome.snu.ac.kr/). Genes were mapped onto chromosome at their genomic position and

95 drawn manually. Duplicated genes were identified by Blast $\mathrm{P}$ search against each other when

96 both their identity and query coverage was $>80 \%$ of their partner sequence [19]. Tandem

97 duplication in genes was identified by occurrence of homologous genes located in single

98 region $(<100 \mathrm{~kb})$ within a chromosome, while segmental duplication occurs among homologous

99 or non-homologous genes with $>1 \mathrm{~kb}$ in length and more than $90 \%$ sequence similarity dispersed

100 but present on same or different chromosomes from the same clade as described by Feng et al.

101 (2017) [20]. Consequently, non-synonymous (Ka) and synonymous substitution (Ks) among

102 duplicated CaRLK gene pairs were calculated using PAL2NAL

103 (http://www.bork.embl.de/pal2nal/). The divergence time of the duplicated gene pairs was

104 calculated as described by Koch et al. (2000) [21].

105 Gene structure and Cis-regulatory element analysis

106 Gene structure was elucidated based on the relationship of the coding sequence and its

107 corresponding genomic DNA sequence by GSDS 2.0 (http://gsds.cbi.pku.edu.cn/).Cis-acting

108 regulatory elements of genomic DNA sequences of $3000 \mathrm{bp} 5$ upstream region was mined from

109 the Sol Genomics Network database. Promoter sequences obtained were submitted in Plant Care

110 Database http://bioinformatics.psb.ugent.be/webtools/plantcare/html/) individually. Conserved

111 biotic-stress responsive elements in hot pepper were predicted, as described by Diao et al. (2018)

$112[22]$.

113 Primer Design 
114 Genomic and its Coding sequences (CDS) of deduced RLK hot pepper proteins were retrieved 115 from Sol Genomics Network (https://solgenomics.net/). Primer sets for RT-qPCR were designed

116 in 3 and 5 untranslated regions of individual genes to avoid non-specific amplification using

117 Prime Quest Tool (http://eu.idtdna.com/PrimerQuest/Home/Index) [23]. For all primers

118 Ubiquitin 3 was used as reference gene. Genes with accession numbers and code assigned in the 119 study (S1-Table).

120 Plant Material, Fungal Strains and Stress Treatments

121 Seeds of two hot pepper genotypes pbc-80 (anthracnose resistant) from National Bureau of Plant 122 and Genetic Resources (NBPGR), Hyderabad and Pusa Jwala (anthracnose susceptible) from 123 Horticulture Research Station, Lam Farm, Guntur, Andhra Pradesh were collected for the study. 124 Seeds were scattered in black trays containing autoclaved blend of peat and vermiculite $(2: 1 \mathrm{v} / \mathrm{v})$ 125 along with micronutrients mixture. Seedlings were raised and watered regularly in a greenhouse 126 under controlled conditions i.e., $16 \mathrm{~h}$ light/ $8 \mathrm{~h}$ dark photoperiod at $27{ }^{\circ} \mathrm{C}$ throughout the day and $12721^{\circ} \mathrm{C}$ during the night. Fungi were isolated from fruit rot infected hot pepper samples by single 128 spore isolation technique. Isolate Colletotrichum truncatum was cultured on Oatmeal agar 129 medium with $\mathrm{pH} 7.0$ at $25 \pm 2^{\circ} \mathrm{C}$. Spore suspension was prepared from seven days old culture and 130 sprayed on seedlings by artificial inoculation method as described by Mishra et al (2017) 131 [24].with $5 \times 10^{5}$ spores/ml concentration. Three-week old hot pepper seedlings were taken for 132 experimental studies. Genotypes PBC-80 and Pusa Jwala sprayed with conidial suspension were 133 considered to be treated and those trays sprayed with autoclaved distilled water as control. Each 134 treatment was maintained with three replications. 
RNA was extracted from leaf tissue of stressed and control genotypes according to acid guanidinium thiocyanate-phenol-chloroform extraction method [25]. Leaf tissue (100 mg) was ground into fine powder by using liquid nitrogen in mortar and pestle. Fine powder was transferred carefully into $2 \mathrm{ml}$ eppendorf tubes with extraction buffer and centrifuged at 12,000 $\mathrm{rpm}$ at $4^{\circ} \mathrm{C}$ for 10 minutes. Supernatant was collected and equal aliquots of chloroform was added to it and centrifuged for 10 minutes at 12,000 rpm. To upper aqueous layer ice cold propanol and $1.5 \mathrm{M} \mathrm{NaCl}$ was added and incubated at $4{ }^{\circ} \mathrm{C}$ for $5 \mathrm{~min}$. Then tubes with solution was centrifuged for 10 minutes at $12,000 \mathrm{rpm}$ and supernatant was discarded. The resultant pellet was subjected to ethanol wash and allowed for air drying. Pellet was dissolved in DEPC water. RNA samples were treated with DNase I (RNase-free) (Takara- cat \# 2270B) to remove residual genomic DNA. The purity and integrity of RNA were determined by calculating A260/A230 and A260/280 absorbance ratio. RNA was visualized in 1.5 percent agarose gel after electrophoresis. Quantitative Real-Time PCR

cDNA was produced in triplicates from three $\mu \mathrm{g}$ of RNA by prime script 1 st strand cDNA synthesis kit (Takara-cat \# 6110A). Gene expression was scrutinized in reverse transcriptionquantitative polymerase chain reaction (RT-qPCR) (QuantStudio3-Applied Biosystems). Twelve $\mu 1$ reaction mixture (Sybr Green $-6 \mu 1, \mathrm{cDNA}-2 \mu 1$, Forward Primer $-1 \mu 1$, Reverse Primer-1 $\mu 1$, DEPC water-2 $\mu 1$ ) was loaded in 96-Well Reaction Plates enclosed with microamp Optical Adhesive Film (Applied Biosystems). Standard cycling parameters and baseline thresholds were set manually along with UBI-3 as a reference gene. CT values were calculated by using software. Proficiency of RT-qPCR reaction for every single RLK gene was deliberated from standard curve gained by serial dilutions of pooled cDNA. Relative gene expression among the samples was calculated using the 2-DDCT method [26]. In this method, an estimated level of 
gene expression was purely based on a hypothesis of $100 \%$ PCR efficiency of reference and target genes. RT-qPCR products were visualized on $2 \%$ agarose gel. The presence of distinct single band with expected amplicon size was considered as specific amplification [27].

\section{Statistical Analysis}

Expression studies for all the four samples (pbc-80 control, pbc- 80 stressed, pusa jwala control and pusa jwala stressed) were carried out at three biological replicates, two technical replicates and were retained at each point of time intervals. Data were represented as mean \pm standard deviation. All the data were tested for significance between resistant and susceptible genotypes using analysis of variance (ANOVA) at 5\% probability. Relative gene expression was generated using heat mapper (http://heatmapper.ca/).

\section{Results}

\section{Identification and annotation of transmembrane receptor kinases}

Blast P homology searches revealed 3,150 hits with pepper genome database. Among them 1,208 hot pepper sequences were found to share more than 50 percent homology with a sequence length ranging from 100-2000 amino acid [28]. From PDB database optimized length of known receptor like kinase sequences ranged from 100-1000 amino acids [S10]. Hence non redundant sequences within the optimized range were considered for further study while remaining redundant sequences were removed manually. A Total of 164 Sequences with typical domain organization viz., presence of signal peptide, varying extracellular domains, a transmembrane region and an intracellular kinase domain were retrieved from respective databases. Based on Conserved motifs type of ectodomain was distinguished and details were illustrated in (S6 Fig). 
181 Naming to each gene was specified with the first two letters indicating Capsicum annuum (Ca)

182

183

184

185

186

187

188

189

190

191

192

193

194

195

196

197

198

199

200

201

202

third letter representing the non-RD class of transmembrane receptor-like kinase (RLK) family followed by serial numbers [29].

\section{Phylogeny Analysis}

Phylogenetic analysis revealed the existence of seven gene families under respective clades in which each clade contains one gene from tomato, and Arabidopsis (Fig 1). Phylogeny grouping revealed the evolutionary relationship of each gene family and their respective subfamilies under respective clades. Pictorial illustration revealed the occurrence of 1. Cysteine-rich receptor-like kinases (Stress antifung) represented in pale green color. 2. Wall Associated Kinases (LRK10L2, LRK10L1) in red color. 3. Ltype Lectin receptor like kinases (lectin Leg B) in violet color. 4. G-type lectin receptor like kinases (SD1a, SD2b, SD3) in green 5. Lys M receptor like kinases in pink color. 6. Malectin receptor like kinases in chocolate brown color. 7. Leucine-rich repeat (LRR) receptor like kinases - LRR Ia, LRR- II, LRR- III, LRR- IV, LRR-V, LRR VIII, LRR IX, LRRXI, LRRXII in dark green color were found. In hot pepper extracellular domains containing RLKs similar to C-type lectin (sky blue), URK1, PERK, extensin (yellow) receptor like kinases were not observed in our analysis.

Fig. 1. Phylogenetic analysis of Receptor like kinase gene family from hot pepper. The phylogenetic tree was constructed using neighbor-joining (NJ) method by MEGA6.0.Subfamilies was specified in different colors

\section{Classification of Kinase Domains}

Based on kinase domain organization CaRLKs were classified into three types. Based on the presence or absence of the conserved residue Arginine (R) present adjacent to Aspartic acid (D) 
203

204

205

206

207

208

209

210

211

212

213

214

215

216

217

218

219

220

221

222

223

224

225

in subdomain VI, conserved lysine (K) residue and Aspartic acid (D) in subdomain II and VII kinase regions. Presence of Arginine (R) adjacent to aspartic acid as RD Kinases those lacking R as Non-RD Kinases and lack of any one or more of the $\mathrm{K} / \mathrm{D} / \mathrm{D}$ residues were classified as $\mathrm{RD}$ minus. Finally, 26 protein sequences were found to possess Leucine, cysteine, phenylalanine, Glycine, and Serine residues substituted in place of arginine. In Supplementary Table (S2) distribution of conserved residues in II, VI and VII domains of 26 genes with respect to their corresponding ectodomain and respective clades fitting to them were given in detail. Multiple sequence alignment of twenty-six genes (S5 Fig) were predicted to share a common linkage as they are descended from a common ancestor. However all 26 CaRLK genes were predominantly grouped under respective LRRXII, LRRXI, SD1a, SD2b, SD3, LRK10L2 and WAK/LRK10L1 clades in their phylogenetic relationship.

\section{Physiochemical Properties}

Non-RD receptor-like protein kinases vary significantly with respect to their structural and physical properties. Genes CaRLK6 with the highest number (1789) of amino acids and CaRLK23 with the lowest number of amino acids (620) was observed while CaRLK17 with high molecular weight (954.89) and CaRLK 23 with low molecular weight (70.11) respectively. Consequently, a wide variation was observed in their isoelectric point (PI) ranging from 5.69 (CaRLK14) to 8.63 (CaRLK10). Instability index ranged from 28.84 - 46.15 where 19 genes were considered to be stable as they exhibit instability index value less than 40 , whereas the rest 7 among 26 non-RD RLKs were considered as unstable. Grand average hydropathicity (Gravy) values ranged from -0.345 (CaRLK19) to +0.12 (CaRLK10) inferring the presence of both hydrophilic and hydrophobic amino acids. Aliphatic index determines the thermal stability of a protein. Among 26 proteins high aliphatic index was observed (CaRLK 19- 79.07 to CaRLK 7- 
111.49) indicating that all are thermally stable with more number of hydrophobic amino acids in their structure. Most of them were found to be localized in extracellular spaces of cell membrane followed by chloroplast, nucleus, and mitochondria. Physiochemical Characteristics of each protein were given (S3 Table) in detail.

\section{Chromosomal Localization}

The inherent ability of a host to defend against pathogens mostly depends on the occurrence of resistance genes and activation of their signaling mechanism present on chromosomes. A total of twenty-six putative genes belonging to non-RD class were aligned on 9 out of 12 chromosomes. Chromosomes 3, 9 and 10 were devoid of non-RD genes, while in contrast a maximum number of seven genes were observed on chromosome 2. Thirteen genes - CaRLK 9,11, 12, 13 on second, CaRLK6, 7 on fourth , CaRLK 2, 8, 10 on sixth while CaRLK3, 4, 5 on fifth and CaRLK 1 on eleventh chromosome belongs to LRRXII subfamily respectively as shown (S4). Single non-RD genes CaRLK 19, 20 and 24 were aligned separately on each of Chromosome 1, 8 and 12 were clustered under SD3, SD2b, and LRK10L2 subfamilies were aligned under same clade respectively. Four genes CaRLK 15, 17, 18, and 21 from same G-type Lectin subfamily located on chromosome 7 were scattered on different clade branches as shown phylogeny figure.

\section{Duplication analysis of CaRLK genes in hot pepper:}

The evolution of gene duplication reveals results in family expansion and the occurrence of novel genes in a genome. Three pairs of genes showed tandem duplication CaRLK 27/28 and CaRLK 13/10, 14/15 while only one paralog pair CaRLK17/18from CRLK was identified to exhibit segmental duplication (S5). All three gene pairs of tandem duplication type possessed $\mathrm{Ka} / \mathrm{Ks}$ ratios less than 0.5 indicating that genes experienced purifying selection pressure. While 
248

249

250

251

252

253

254

255

256

257

258

259

260

261

262

263

264

265

266

267

268

269

gene pair CaRLK $17 \& 18$ showed $\mathrm{Ka} / \mathrm{Ks}$ ratios $<1.0$ implying positive mode of selection. The divergence time of non-RD genes exposes the duplication events started from 5.86 Mya and continued up to 3.77 Mya in evolution.

\section{Gene structure analysis}

Gene length varied from 2,224 bp (CaRLK 24) to 21,556 bp (CaRLK 6). Moreover, genes with either positive or negative sense strand as a template to the coding regions are depicted in Supplementary Table (S3). Various exon-intron positions were compared to gain insight into possible mechanisms of structural diversity existing among Non-RD kinases in Capsicum annuum. In this study introns varied from 0-12 in number. CaRLK 6 gene from LRRXII family showed a maximum of 12 introns. While in contrast a total of four genes (CaRLK 17, 18, 20 and 21) from G- type lectin and single gene CaRLK 13 from LRR type family were found to occur without introns in their structure. Genes from same family showed similar intron organization. Where Genes CaRLK 15, 16 from Stress-antifung subfamily showed six number of introns. While contrarily genes from LRR family possessed varied introns like ten genes (CaRLK 1, 2, 3, 4, 5, 8, 9, 14 ,19 and 23) with single intron. Four Genes (CaRLK 10, 11, 22 and 24) with two introns and three genes (CaRLK 7, 25, 26) with three introns were observed in their structural organization. Moreover LRK10L2 clade members CaRLK23 with single intron and CaRLK 22, 24 with two introns also showed varied introns organization in their structure

\section{Cis-regulatory element analysis}

Cis-regulatory element analysis promotes a good insight to understand the expression patterns of a gene under various stress conditions, whose validation needs to be warranted. Major pathogeninduced cis-regulatory elements identified in hot pepper (S7). Among twenty-six non RD genes 
270 highest number of cis-regulatory elements (TGACG, STRE) known to be involved in defense

271 and stress responses were found in CaRLK 3, 11 from LRR and CaRLK 16 from G-type lectin

272 subfamily. Fungal elicitor and oxidative responsive cis regulatory elements viz., W-box, F-box,

273 As1 and box4 were observed majorly in CaRLK 1, 7,8 from LRR and 22, 26 from WAK family

274 Correspondingly elicitor responsive element G-box and Abscisic acid signifying region ABRE

275 were observed in abundance among promoter regions of CaRLK3, 15, 17, 23 and 26 genes.

276 Whereas Myb and Myc binding sites responsible for triggering stress-responsive metabolic

277 pathway were found to occur predominately in CaRLK21, CaRLK 1 and 15. Moreover, only 32

278 percent of genes showed the presence of TC-rich repeat and TCA element linked with salicylic

279 acid and methyl jasmonic acid pathway. When compared to all genes CaRLK1 and CaRLK16

280 from LRR family were identified to hold more number of cis-regulatory elements and may

281 associate intensely with defense responsive mechanism.

\section{Heat Map Analysis}

283 Differential expression of three family genes at varied time interval was observed in resistant 284 (pbc-80) and susceptible (PJ) Hot pepper genotypes (Fig 2).

285 Fig. 2. Differential expression analyses of CaRLK genes under biotic stress. Treatment in PBC-

28680 (a) and pusa jwala (b) Hot pepper seedlings. The color scale represents log2 expression

287 values.

288 Expression profiles of CaRLK genes at various stress treatments

289 At Initial stages of infection after 24 hours of inoculation, genes from LRR type (CaRLK 14, 1)

290 G-type lectin (CaRLK15, 16 and 19) and Wall associated kinase (CaRLK 23, 24) showed 291 significant up-regulation in Pbc-80 when compared to that of Pusa jwala cultivar (Fig 3.a). At 
292 biotrophic phase of infection, during colonization of subcuticular hyphae beneath the cuticle in

293 resistant when compared to that of susceptible genotype after 48 hours of inoculation (Fig 3.b).

294 Genes from $\operatorname{LRR}(14,6,3,1,2,9,10)$, G-type lectin $(16,17,18,19)$ and WAK $(23,24,26)$ families

295 showed significant up-regulation while LRR (CaRLK2) exhibited down-regulation. During the

296 necrotic phase, after 72 hours of inoculation defense-related genes (Fig 3.c). Gene CaRLK 2

297 from G-Lectin family, CaRLK 23, 25 from WAK, CaRLK 1, 9 and 14 from LRR families

298 showed significant up-regulation. Furthermore genes CaRLK 17, 18 from G type lectin and

299 CaRLK7 from LRR family have shown down regulation in resistant genotype when compared to 300 susceptible genotype at a phase where extensive cell death of epidermal and mesophyll cells,

301 instigating necrotic phase. In the present investigation number of genes belonging to LRR family

302 was up-regulated in Pbc-80 indicating a potential defensive role. At formation of acervullus stage

303 (216h after inoculation), genes from $\operatorname{LRR}(6,7,11,1,2,14)$, WAK $(22,23,24,25,26)$ and G-Lectins

$304(15,16,19,20)$ were significantly up-regulated While CaRLK 7,17 and 22 were down-regulated in 305 pbc-80 when compared to pusajwala (Fig 3.d). Instability index of all genes up-regulated 306 remained unstable in nature. At last stage, conidial dispersion after $216 \mathrm{~h}$ of inoculation. Genes 307 from LRR family CaRLK 6, 1 and from LRRXII subfamily CaRLK8 showed a significant up308 regulation. Whereas LRR (CaRLK 6) has down-regulated at severe stress conditions (Fig 3.e).

309 Fig 3. Expression profiles of CaRLK genes in leaf tissue in response to Colletotrichum 310 truncatum at various time intervals (a) 24, (b) 48, (c) 72, (d) 148 (e) 216 hours after inoculation. 311 Mean values and Standard Deviation for three replicates are shown.

\section{Discussion}


313 Receptor-like kinases plays a vital role in plant development, signal transduction and defense

314 responses [30]. Availability of sequenced genomes had facilitated the researchers to study

315 various functional roles of RLK family genes in various stress adaptation procedures in many

316 model plants like Rice, Arabidopsis, Tobacco, Wheat, Tomato, Soybean etc., [31]. Most of the

317 candidate RLK genes involved in primary immune responses associated with disease resistance

318 belong to non-RDclass kinases. Present investigation was aimed at genome-wide identification

319 of non-RD kinases in hot pepper a vegetable crop with global agricultural and economic

320 importance, whose production has been hindered by several biotic stresses [32]. A total of 8 and

32135 percent of non-RD motifs were identified among IRAK family members in Arabidopsis and

322 Rice [7]. This huge difference may be due to monocot-dicot diversification. Comparative

323 phylogeny of hot pepper with model plants revealed the evolutionary existence of seven

324 subfamilies from non RD class of RLK gene family. CaRLK genes were more closely related to

325 genes from arabidopsis and tomato than rice, reflecting the fact that arabidopsis, tomato and

326 pepper are eudicots and diverged more recently from a common ancestor [33].

Gene duplication events majorly include tandem, segmental and whole-genome

328 duplications with substantial roles occurring in the evolution [34]. In hot pepper among 26 genes

329 three pairs from chromosome 2 and 11 unveiled tandem duplications within LRR and WAK

330 families evolved from common ancestor LRRXII family. In hot pepper, tandem duplication may

331 signify LRRXII family lineage-specific expansion with novel gene expression. Whereas

332 Hofberger et al. (2015) reported lineage-specific expansion of L-type lectin receptor kinase gene

333 family by tandem duplication event in Brassicaceae. While only one pair from the stress-antifung

334 subfamily of G-type lectin showed segmental duplication in the second chromosome. Segmental

335 duplication majorly contributes to gene expression and plays an important role in immunity, 
growth and defense responses to external stimuli [20]. Results were in accordance with Cannon et al. (2004) [35] who reported a negative correlation between tandem and segmental duplication within Arabidopsis gene families. Furthermore, the substitution rate of non-synonymous (Ka) and synonymous (Ks) mutations was assessed to evaluate the selection pressures and divergence time succeeded among the duplicated CaRLK gene pairs (S4). LRR and WAK gene families speciation or adaptation. While single gene pair G-type lectin family showed positive selection and may be involved in functional diversification as described by Haung et al. (2016) [36]. or gene expression regulation [37]. Five intron less genes were found in hot pepper, likewise,

346 Yang et al. (2009) [38] also reported the presence of intron less genes in taxonomic species like 347 Arabidopsis thaliana, Populusdeltoides and Oryza sativa representing their lineage-specific 348 expansion with specific function in the evolution. In hot pepper among 26 non-RD genes only 10 349 genes had exhibited single intron in its structure. Xu et al. (2017) [34] also reported the presence 350 of fifteen single intron genes in Populus deltoides with multiple functions. Genes present in a 351 family usually contain the same structural organization but conversely, some genes from SD3, LRK10L2, and LRRXII subfamilies showed varied intron-exon organization in hot pepper.

353 These variations may be due to substituted residues in conserved positions depicting the 354 evolutionary changes occurring within a family [39]. Cis-regulatory elements are noncoding sequences that act as binding sites for transcription factors involved in proper spatiotemporal expression of genes containing them. In 357 rice pathogen-induced, cis-regulatory elements like AS-1, G-box, GCC-box, and H-box were expressed and confirmed as markers for identification of resistance genes in response to fungal 
359 infection (Kong et al., 2018). While few genes CaRLK 17 and 3 from SD3 and LRRXII

360 subfamily were found to possess those genes and may also imply the same function. The

361 presence of Cis-regulatory elements like F/Sbox, W box, TGACG and MYB binding site in

362 promoter regions are involved in the stress-inducible defense gene in Maize [40].

In this investigation, we had analyzed differential expression patterns of particular gene

364 during various temporal stages based on disease progression studies (Fig 4). Infection stages of

365 Colletotrichum truncatum like spore adhesion, germination, and penetration by appressorium

366 (24h), subcuticular colonization of hyphae (48h), aggregation of mycelium (72h), Acervullus

367 formation (148h), Conidial dispersion (216h) after inoculation on leaf surface of hot pepper

368 seedlings was observed. Gene CaRLK 1 showed the highest expression in response to

369 Colletotrichum truncatum even after 216hours of infection. Our results were in accordance with

370 Sakamoto et al. (2012) who reported the significance of LRRXII family genes in providing

371 resistance against necrotic fungi in tomato genotype. In hot pepper three genes (CaRLK23, 24

372 and 25) from subfamily LRK10L2, two genes (CaRLK15, 16) from SD1a subfamily and CaRLK

37325,26 from WAKLRK10L1 had showed significant up-regulation in resistant variety

374 particularly during hyphae colonization and acervuli formation. Presence of cis-regulatory

375 elements like TGACG (MeJA responsive), ERE (Ethylene stress responses) STRE, MYB and

376 MYC (defense responsive) in promoter region are majorly involved in JA-ET pathway may be

377 responsible for providing resistance. Chowdhury et al. (2017) [41]. Reported an increase in

378 Jasmonic Acid (JA) and Ethylene (ET) hormone-mediated signaling pathways during biotrophic

379 and necrotrophic phase of Colletotrichum infection which was responsible for governing disease

380 resistance in sesame. Three genes CaRLK 17, 18, 19 from SD3 subfamily with cis-regulatory

381 element like TCA element involved in salicylic acid regulation had showed significant up- 
382 regulation only during hyphae colonization. Qi et al. (2012) [42] reported the role of SA in

383 hyphae growth and basal defense response. While Genes from SD2b family didn't exhibit any

384 up-regulation. Few genes CaRLK 2, 7, 22 and 17 from various subfamilies had showed

385 significant downregulation. In rice among four WAK members, three genes OsWAK14,

386 OsWAK91 and OsWAK92 act as positive regulators and OsWAK112d as negative regulator

387 while providing quantitative resistance against magnaporthae oryzae [43]. Expression studies

388 by Rt-qPCR were used to identify candidate genes / functional markers majorly involved in crop

389 improvement programs.

390 Fig. 4. : Histochemical observation of Colletotrichum truncatum infective structures on hot

391 pepper leaves under electron microscope at $40 \mathrm{X}$ magnification.

392 Conclusion

393 This is the first report of genome-wide identification, characterization, and expression profiling

394 of the non-RD kinase gene family in hot pepper. We had systematically analyzed and identified

$39526 \mathrm{CaRLKs}$, and characterized those using bioinformatics and expression analyses in response to

396 Colletotrichum truncatum stress conditions. After nine days of infection one gene (CaRLK1)

397 from LRRXII subfamily out of 26 non-RD genes was found to be expressed more in resistant

398 genotype (PBC-80) than in susceptible genotype (Pusajwala). Moreover, identification of cis-

399 elements in this gene enabled us to understand their role in conferring resistance in PBC-80

400 genotype by activation of the phytohormone JA-ET signaling pathway. Therefore this

401 comprehensive analysis serves as a central platform to understand various physiological and

402 biochemical functions performed by CaRLK1 gene in providing resistance. This investigation

403 can also pave a way to analyze stress responsive gene expression studies in various pepper 
404 tissues and aids in promoting sustainable agriculture by using different crop improvement

405 methods

\section{Acknowledgment}

407 Authors are thankful to departmental heads and Co-scholars of bioinformatics lab for providing

408 facilities to carry out the work.

409 Supplementary data

410 All data associated with this paper can be found within the supplementary files.

411 References

412 1. Ridzuan R, Rafii MY, Ismail SI, Mohammad Yusoff M, Miah G, Usman M. Breeding for 413 anthracnose disease resistance in chili: progress and prospects. International journal of $414 \quad$ molecular sciences. 2018 Oct;19(10):3122.

2. Saxena A, Raghuwanshi R, Singh HB. Molecular, phenotypic and pathogenic variability in $\mathrm{C}$ olletotrichum isolates of subtropical region in north-eastern India, causing fruit rot of chillies. Journal of applied microbiology. 2014 Nov;117(5):1422-34.

3. Roy CB, Liu H, Rajamani A, Saha T. Transcriptome profiling reveals genetic basis of disease resistance against Corynespora cassiicola in rubber tree (Hevea brasiliensis). Current Plant Biology. 2019 Jan 1;17:2-16.

4. Saijo Y, Loo EP, Yasuda S. Pattern recognition receptors and signaling in plant-microbe interactions. The Plant Journal. 2018 Feb;93(4):592-613. of botany. 2004 Jan 1;82(1):1-5. 
6. Dardick C, Ronald P. Plant and animal pathogen recognition receptors signal through non-RD kinases. Plos pathogens. 2006 Jan 20;2(1):e2.

7. Dardick C, Schwessinger B, Ronald P. Non-arginine-aspartate (non-RD) kinases are associated with innate immune receptors that recognize conserved microbial signatures. Current opinion in plant biology. 2012 Aug 1;15 (4):358-66.

8. Zipfel C, Kunze G, Chinchilla D, Caniard A, Jones JD, Boller T, Felix G. Perception of the bacterial PAMP EF-Tu by the receptor EFR restricts Agrobacterium-mediated transformation. Cell. 2006 May 19; 125(4):749-60.

9. Singh P, Kuo YC, Mishra S, Tsai CH, Chien CC, Chen CW, Desclos-Theveniau M, Chu PW, Schulze B, Chinchilla D, Boller T. The lectin receptor kinase-VI. 2 is required for priming and positively regulates Arabidopsis pattern-triggered immunity. The Plant Cell. 2012 Mar 1;24 (3):1256-70.

10. Bai Y, Sunarti S, Kissoudis C, Visser RG, Van Der Linden G. The role of tomato WRKY genes in plant responses to combined abiotic and biotic stresses. Frontiers in plant science. 2018;9:801.

11. Petersen TN, Brunak S, Von Heijne G, Nielsen H. Signalp 4.0: discriminating signal peptides from transmembrane regions. Nature methods. 2011 Oct;8(10):785.

12. Letunic I, Doerks T, Bork P. SMART 7: recent updates to the protein domain annotation resource. Nucleic acids research. 2011 Nov 2;40(D1):D302-5. 
13. Bateman A, Birney E, Cerruti L, Durbin R, Etwiller L, Eddy SR, Griffiths-Jones S, Howe KL, Marshall M, Sonnhammer EL. The Pfam protein families database. Nucleic acids research. 2002 Jan 1;30(1):276-80. 2015 May 7;43(W1):W39-49. RLK superfamily: phylogeny and functional predictions about the role of the LRRII-RLK signaling. Sci. STKE. 2001 Dec 18;2001(113):re22-.

17. Tamura K, Stecher G, Peterson D, Filipski A, Kumar S. MEGA6: molecular evolutionary genetics analysis version 6.0. Molecular biology and evolution. 2013 Oct $16 ; 30(12): 2725-9$. chitin oligosaccharides contributes to disease resistance to blast fungus Magnaporthe oryzae in rice. The Plant Journal. 2010 Oct;64(2):343-54. genome-wide segmental duplications reveals a common genomic feature of association with immunity among domestic animals. BMC genomics. 2017 Dec;18(1):293. 
20. Koch MA, Haubold B, Mitchell-Olds T. Comparative evolutionary analysis of chalcone synthase and alcohol dehydrogenase loci in Arabidopsis, Arabis, and related genera (Brassicaceae). Molecular biology and evolution. 2000 Oct 1;17(10):1483-98. analyses of the NAC transcription factor gene family in pepper (Capsicum annuum L.): chromosome location, phylogeny, structure, expression patterns, cis-elements in the promoter, and interaction network. International journal of molecular sciences. 2018

22. Bárzana G, Aroca R, Bienert GP, Chaumont F, Ruiz-Lozano JM. New insights into the regulation of aquaporins by the arbuscular mycorrhizal symbiosis in maize plants under drought stress and possible implications for plant performance. Molecular Plant-Microbe Interactions. 2014 Apr;27(4):349-63.

23. Mishra R, Nanda S, Rout E, Chand SK, Mohanty JN, Joshi RK. Differential expression of defense-related genes in chilli pepper infected with anthracnose pathogen Colletotrichum truncatum. Physiological and molecular plant pathology. 2017 Jan 1;97:10. thiocyanate-phenol-chloroform extraction: twenty-something years on. Nature protocols. quantitative PCR and the 2- $\Delta \Delta \mathrm{CT}$ method. Methods. 2001 Dec 1;25(4):402-8. 
26. Hachez C, Moshelion M, Zelazny E, Cavez D, Chaumont F. Localization and quantification of plasma membrane aquaporin expression in maize primary root: a clue to understanding their role as cellular plumbers. Plant molecular biology. 2006 Sep 1; 62 (12):305-23.

27. He S. Genome-wide identification and transcriptional expression analysis of mitogenactivated protein kinase and mitogen-activated protein kinase kinase genes in Capsicum annuum. Frontiers in plant science. 2015 Sep 25;6: 780.

28. Qin L, Mo N, Muhammad T, Liang Y. Genome-wide analysis of DCL, AGO, and RDR gene families in pepper (Capsicum annuum L.). International journal of molecular sciences. 2018 Apr;19(4):1038.

29. Afzal AJ, Wood AJ, Lightfoot DA. Plant receptor-like serine threonine kinases: roles in 495 signaling and plant defense. Molecular Plant-Microbe Interactions. 2008 May;21(5):507-

30. Passricha N, Saifi SK, Singh R, Kharb P, Tuteja N. Receptor-Like Kinases Control the Development, Stress Response, and Senescence in Plants. Insenescence Signalling and Control in Plants 2019 Jan 1 (pp. 199-210). Academic Press.

31. Saxena A, Raghuwanshi R, Gupta VK, Singh HB. Chilli anthracnose: The epidemiology and management. Frontiers in microbiology. 2016 Sep 30;7:1527. identification and analysis of the SBP-box family genes in apple (Malus $\times$ domestica Borkh.). Plant Physiology and Biochemistry. 2013 Sep 1;70:100-14. 
33. Xu Z, Gao L, Tang M, Qu C, Huang J, Wang Q, Yang C, Liu G, Yang C. Genome-wide identification and expression profile analysis of $\mathrm{CCH}$ gene family in Populus. Peerj. 2017 Oct 27;5:e3962.

34. Hofberger JA, Nsibo DL, Govers F, Bouwmeester K, Schranz ME. A complex interplay of tandem-and whole-genome duplication drives expansion of the L-type lectin receptor kinase gene family in the brassicaceae. Genome biology and evolution. 2015 Jan $28 ; 7(3): 720-34$.

35. Cannon SB, Mitra A, Baumgarten A, Young ND, May G. The roles of segmental and tandem gene duplication in the evolution of large gene families in Arabidopsis thaliana. BMC plant biology. 2004 Dec;4 (1):10.

36. Huang $\mathrm{BH}$, Chen YW, Huang CL, Gao J, Liao PC. Imbalanced positive selection maintains the functional divergence of duplicated DIHYDROKAEMPFEROL 4REDUCTASE genes. Scientific reports. 2016 Dec 14; 6:39031.

37. Wang Y, Liu J, Huang BO, Xu YM, Li J, Huang LF, Lin J, Zhang J, Min QH, Yang WM, Wang XZ. Mechanism of alternative splicing and its regulation. Biomedical reports. 2015 Mar 1;3(2):152-8.

38. Yang X, Jawdy S, Tschaplinski TJ, Tuskan GA. Genome-wide identification of lineagespecific genes in Arabidopsis, Oryza and Populus. Genomics. 2009 May 1;93 (5):473-80.

39. Liu PL, Huang Y, Shi PH, Yu M, Xie JB, Xie L. Duplication and diversification of lectin receptor-like kinases (lecrlk) genes in soybean. Scientific reports. 2018 Apr 12;8(1):5861. 


\section{Supporting information}

S4 Table. Estimated $\mathrm{Ka} / \mathrm{Ks}$ ratios and divergence times of the duplicated CaRLK genes

542 S5 Fig. Multiple sequence alignment of conserved motifs in kinase region of Hot pepper

543 S6 Fig. Identified conserved motifs in non-RD RLK in hot pepper.

40. Jin B, Sheng Z, Muhammad I, Chen J, Yang H. Cloning and functional analysis of the promoter of a stress-inducible gene (Zmap) in maize. Plos one. 2019 Feb 8; 14(2):e0211941.

41. Chowdhury S, Basu A, Kundu S. Biotrophy-necrotrophy switch in pathogen evoke differential response in resistant and susceptible sesame involving multiple signaling pathways at different phases. Scientific reports. 2017 Dec 8;7 (1):17251.

42. Qi PF, Johnston A, Balcerzak M, Rocheleau H, Harris LJ, Long XY, Wei YM, Zheng YL, Ouellet T. Effect of salicylic acid on Fusarium graminearum, the major causal agent of fusarium head blight in wheat. Fungal Biology. 2012 Mar 1; 116 (3):413-26.

43. Delteil A, Gobbato E, Cayrol B, Estevan J, Michel-Romiti C, Dievart A, Kroj T, Morel JB. Several wall-associated kinases participate positively and negatively in basal defense against rice blast fungus. BMC plant biology. 2016 Dec; 16(1):17.

S1 Table. List of primers used for RLK expression analysis

S2 Table. Conserved motif based identification of Non RD kinases

S3 Table. Physiochemical properties of non-RD kinases in Hot pepper

S7 Fig. Distribution of non-RD kinases on hot pepper chromosomes. Chromosome numbers are indicated at top of each bar. Scale is represented in mega bases (Mb). 
546 S8 Fig. Exon-intron structure of non-RD kinases.

547

Yellow indicates exon, black line indicates intron.

548 S9 Fig. Cis-acting elements in the promoter regions of CaRLK genes.

549 S10 Fig. Sequence length cutoffs to build the credible set 
Figures

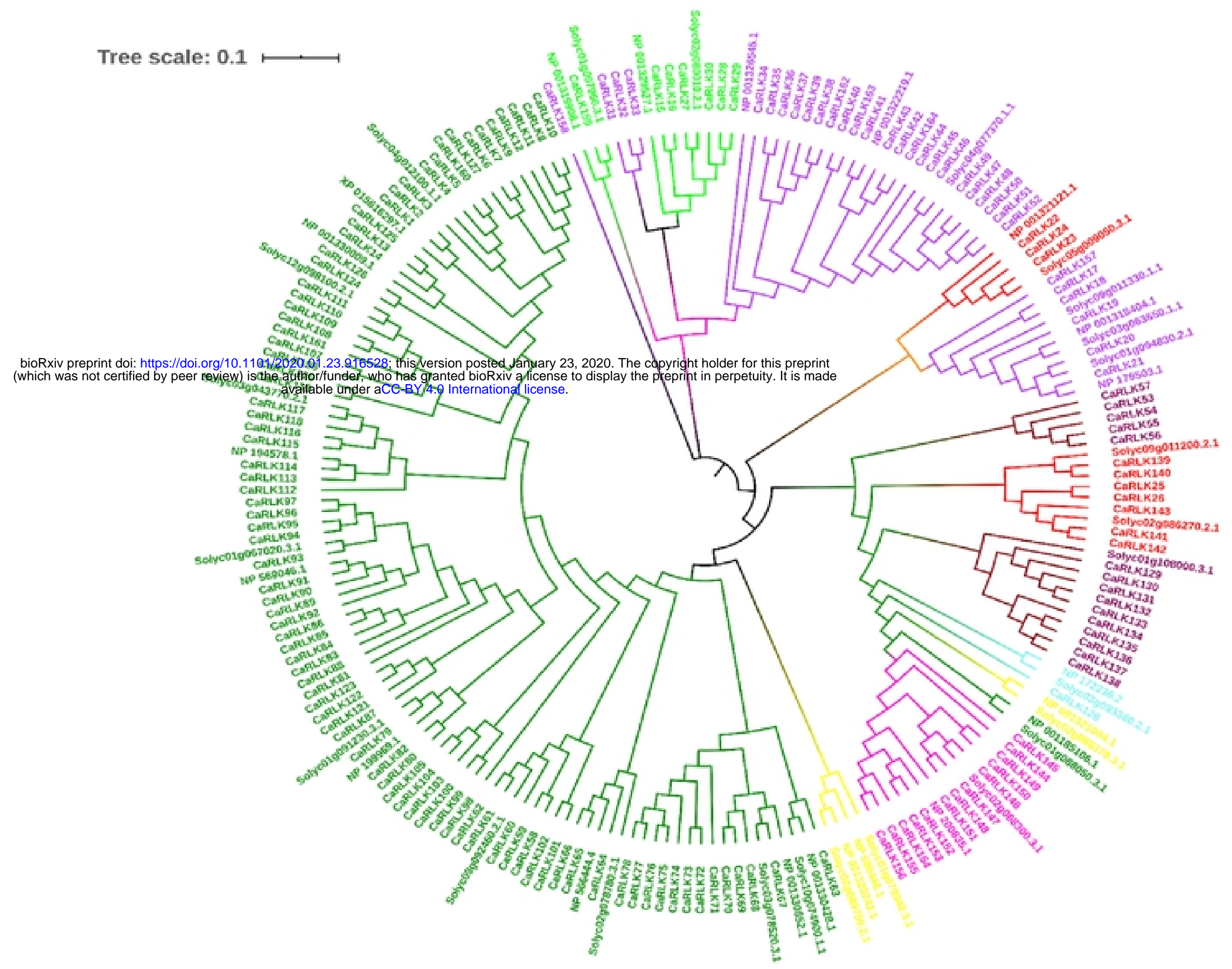

Fig. 1. Phylogenetic analysis of Receptor like kinase gene family from hot pepper. The phylogenetic tree was constructed using neighbor-joining (NJ) method by MEGA6.0. Subfamilies was specified in different colors. 
(a)

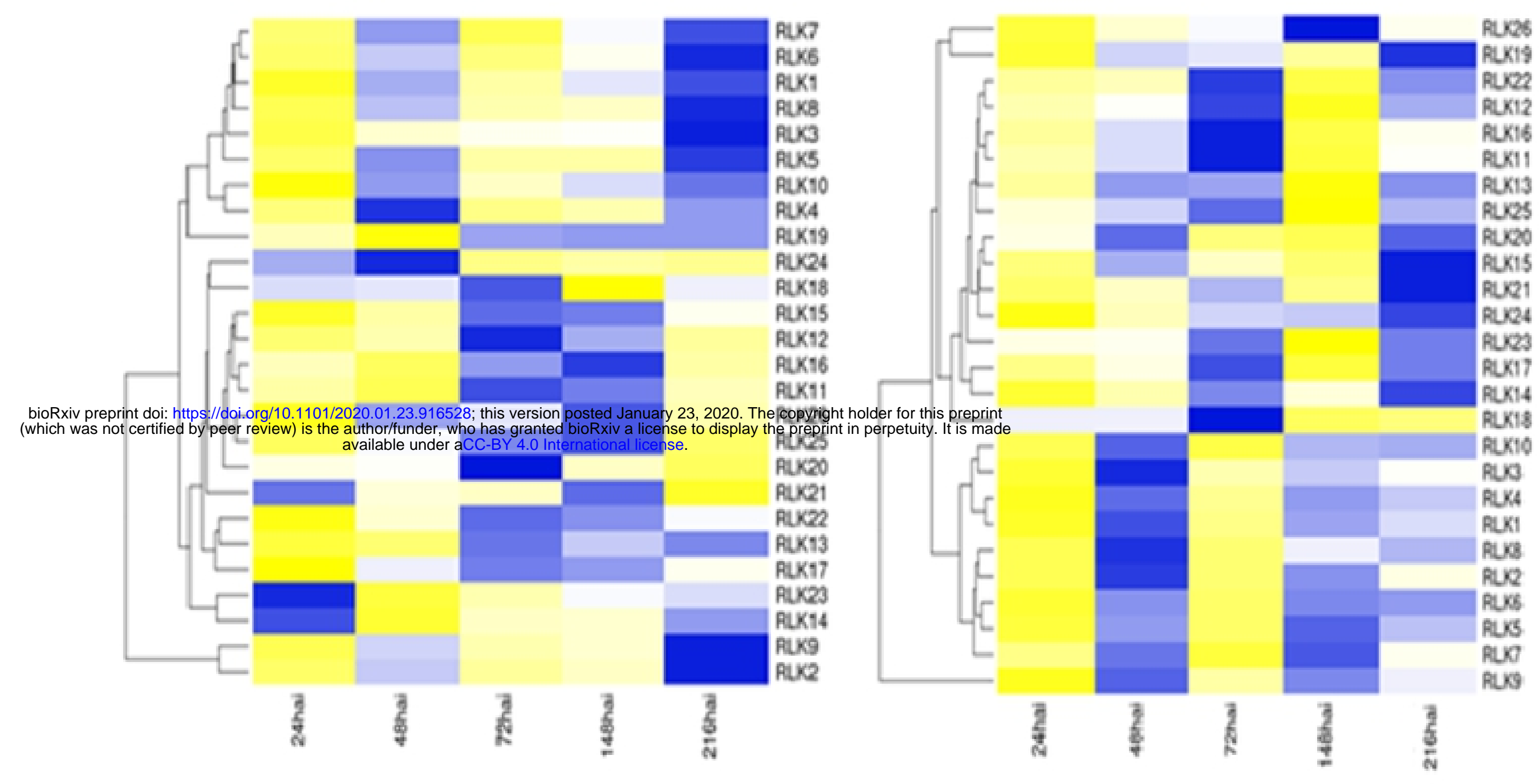

Fig. 2. Differential expression analyses of CaRLK genes under biotic stress. Treatment in PBC80 (a) and pusa jwala (b) Hot pepper seedlings. The color scale represents log2 expression values. 


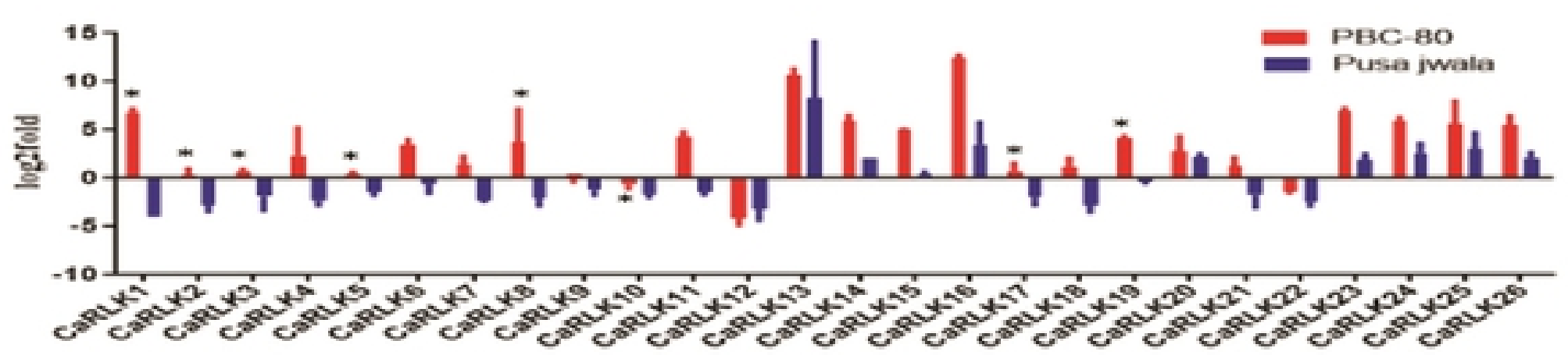

(a) CaRLKGenes
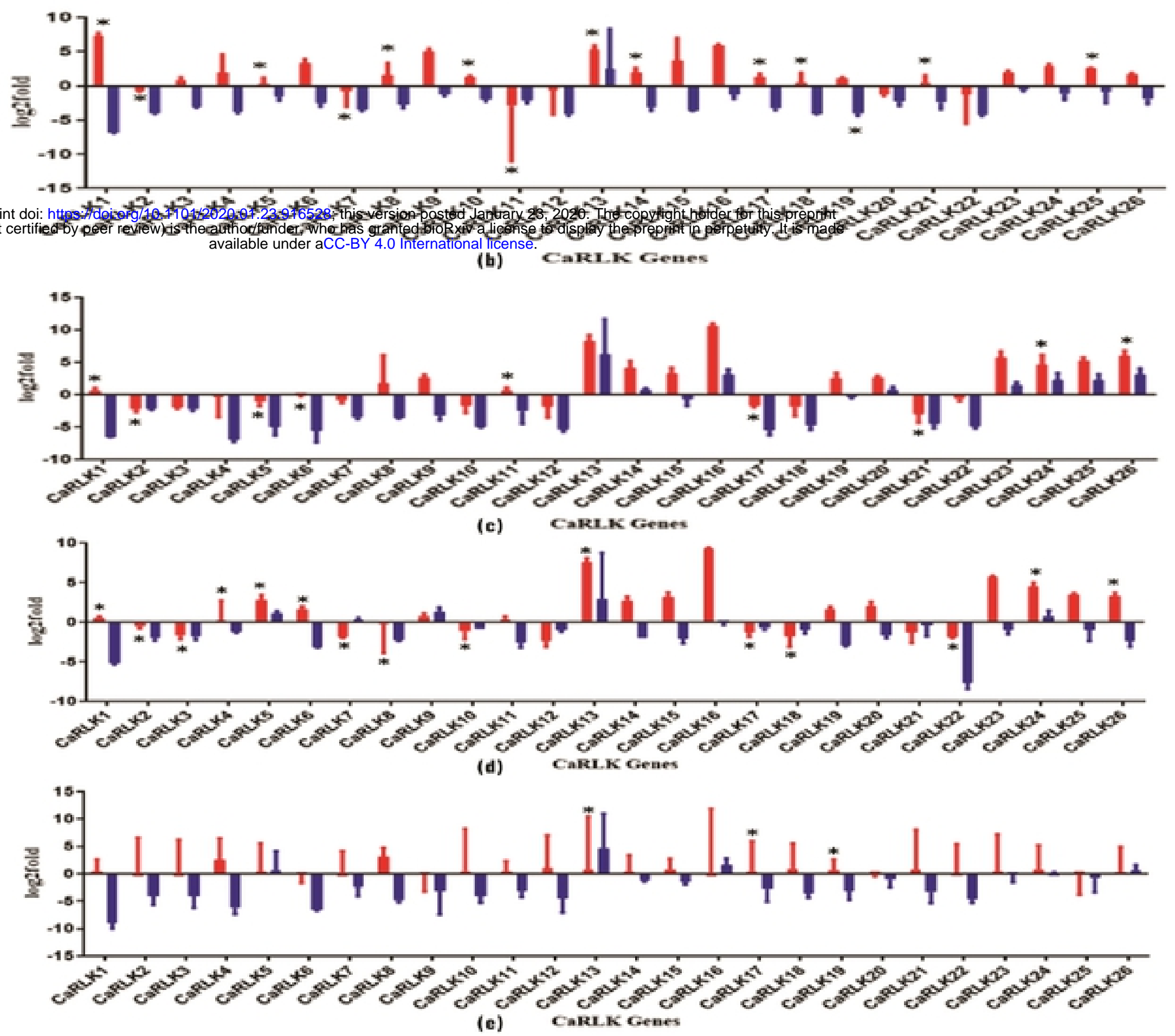

Fig 3. Expression profiles of CaRLK genes in leaf tissue in response to Colletotrichum truncatum at various time intervals (a) 24, (b) 48, (c) 72, (d) 148 (e) 216 hours after inoculation. Mean values and Standard Deviation for three replicates are shown. 

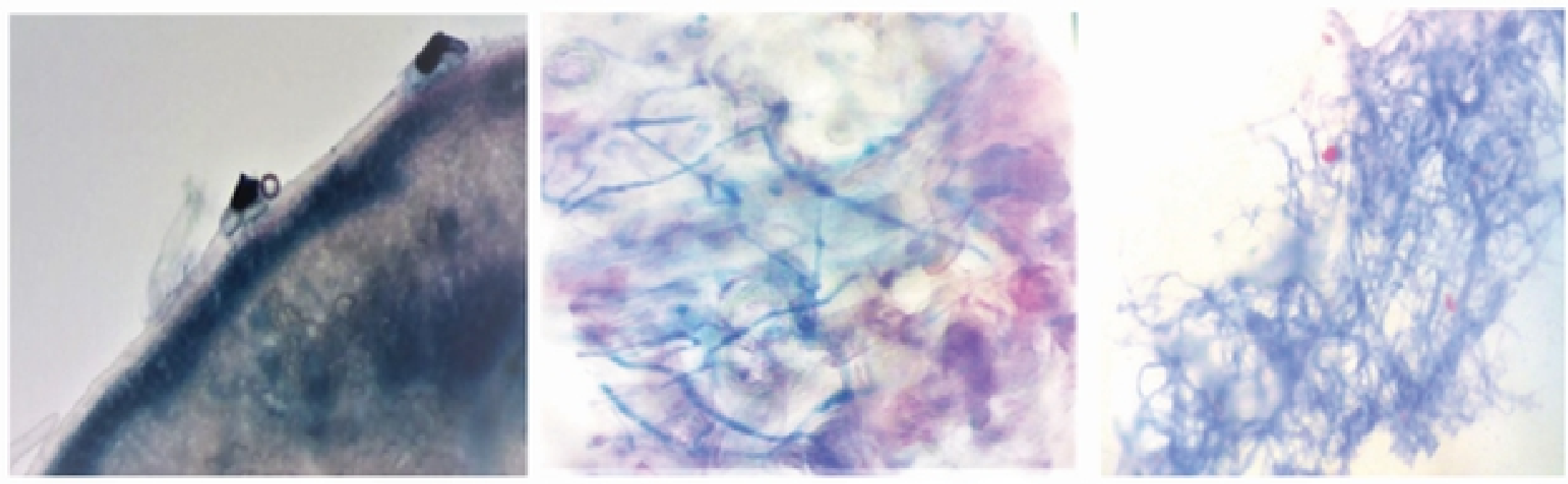

Appresorium penetration (24-hpi)
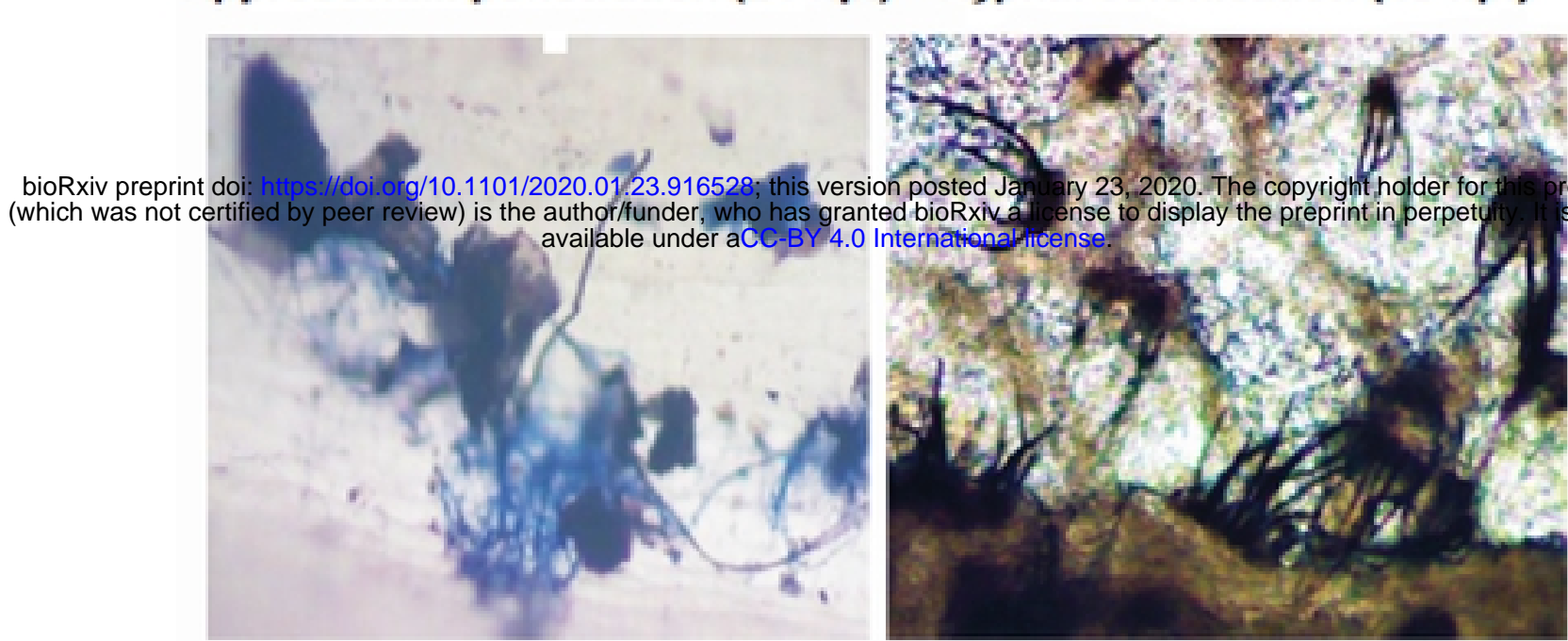

Mycelial aggregation (72-hpi)

Acervulli formation

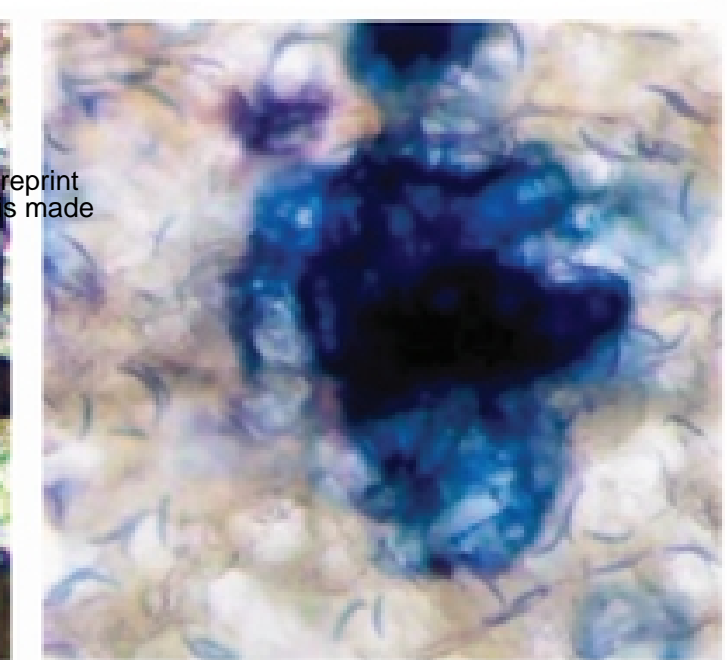

Acervuli-setae formation (148-hpi) Conidial dispersion (216-hpi)

Fig. 4. : Histochemical observation of Colletotrichum truncatum infective structures on hot pepper leaves under electron microscope at $40 \mathrm{X}$ magnification. 